\title{
A Minimum Machining Cost Approach for Automated Tolerance Assignment in Concentricity Control
}

\author{
Chun-Wei R. Lin and Yuh-Jiuan M. Parng
}

\begin{abstract}
Location is a commonly used tolerance control criteria in the geometric dimensioning and tolerancing area. This tolerance is of special importance to the high precision assembly industry. The control of tolerance in design, manufacturing, and assembly is vital to preserving the functionality of products, the validity of assembly plans, and the optimality of the tolerance and equipment assignment. This research develops a computer-aided assembly process tolerance control system to help the assembly process planner to verify the tentative assembly process plan. Also, a weighted assembly process decision support module is incorporated to help adjusting the tolerance allocation as well as the selection of appropriate assembly procedures. The optimality of the resultant assembly plan is ensured based on a minimum cost model by using the mixed integer programming technique.
\end{abstract}

Index Terms-Machining cost control, tolerance control, process planning.

\section{INTRODUCTION}

Tolerance control in design, manufacturing, and assembly is vital to preserving the functionality of products, the validity of assembly plans, and the optimality of the tolerance and equipment assignment. Especially, the location tolerance is a common critical control issue for the assembly processes. A computer aided tolerance control module should be integrated with other modules in a computer-aided design and manufacturing (CAD/CAM) system and served as a intelligent post processor for a computer aided process planning system (CAPP).

Such tolerance control module can identify the assembly process significance corresponding to each design dimension and tolerance specification. The assembly process planner can pay attention only to those critical operations which dominate the resultant tolerance of the tentative assembly plan. Also, by incorporating optimization procedures into the tolerance control module, the process planner can select equipment with more appropriate capability and adjust the tolerance assignment for each critical operation.

In order to maintain the completeness of the tolerance control module, it is critical for such module to be able to handle both linear and geometric dimensional tolerances. However, most of the existing literatures provide only the treatment for linear dimensional tolerances [1]. Only few researchers have covered geometric dimensional tolerances, such as position and form tolerances which are important for

Manuscript received November 9, 2016; revised February 27, 2017.

Chun-Wei R. Lin is with Business Administration Department, Asia University, Wufeng 41354, Taiwan, R.O.C. (e-mail: lincwr@ asia.edu.tw).

Yuh-Jiuan M. Parng is with Accounting Department, Asia University, Wufeng 41354, Taiwan, R.O.C. (e-mail: melodyp@ asia.edu.tw). assembly and hole making processes [2].

In this paper, the analysis and control, for concentricity of location tolerance is fully explored. Then the algorithm of constructing the tolerance chart for concentricity is developed. Such algorithm also demonstrates the feasibility to automate the generation for location and orientation tolerance charts. Also, a decision support module based on the weighted assembly operation vs. machine selection factor is generated. A minimum cost model by using mixed integer programming technique is created as well. Lastly, the implementation of a computer-aided assembly process tolerance control system is presented.

\section{LINEAR TOLERANCE CHART}
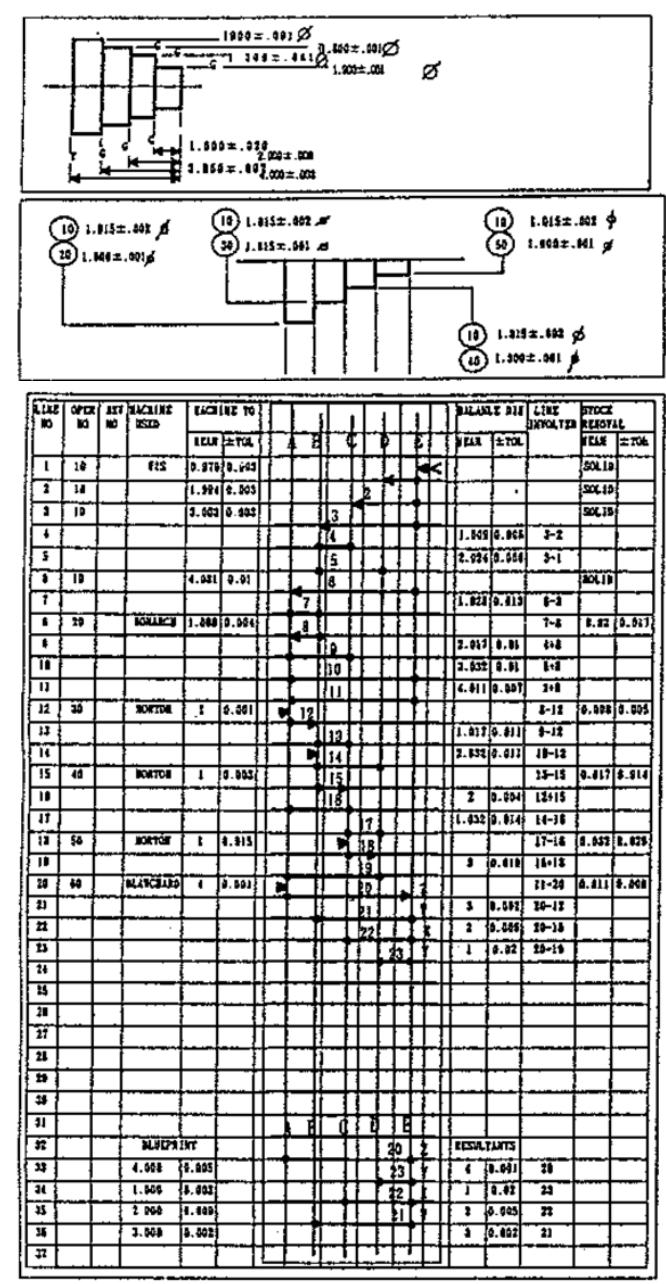

Fig. 1. Lonear Tolerance Chart [3].

A linear tolerance chart example for calculating resultant linear dimensions and tolerances is given in Fig. 1. Basically, three types of important information are conveyed in the 
linear tolerance chart. First, the information about process capability is given in the column of working dimension and tolerance. Second, the intermediate part dimension and tolerance information can be obtained from the column of dimension and tolerance for stock removal. Third, both of the $\mathrm{B} / \mathrm{P}$ specification and the resultant tolerances are summarized in the lower part of the tolerance chart.

Two situations acquire modification for the intended process plan: 1). If the range of a resultant tolerance is looser than the value specified on the $\mathrm{B} / \mathrm{P}$, then the process planner should select machines with higher capabilities for some of the critical operations, or 2) If the intended process plan produces tighter resultant tolerance than required, then the tolerance for some of the critical operations can be relaxed. Detailed algorithms for constructing a linear tolerance chart can be found in Irani et al. [3].

\section{LITERATURE REVIEW}

In order to provide solid theoretical foundations for computer-aided assembly process tolerance control system, the literature review of this research are carried out in the following three categories: 1) Functional requirements of a computer-aided tolerancing system, 2) Modeling and control of geometric tolerances, and 3) Optimal tolerance assignment:

\section{A. Functional Requirements of a Computer-Aided Tolerance System}

Germani et al. [4] have suggested basic stages for a computer based tolerance analysis control system: 1) Describing and quantifying the functional requirements of the design, 2) Identifying datum, features, and geometric relations between the features, 3) Developing functional equations that the function requirements are defined as dependent variables; and, the design sizes and tolerances of the dimensions affecting the functional requirements are defined as independent variables, and 4) Determining an economic solution to these equations.

Haghighi et al. [1] have developed an automated tolerance stack detection software for mechanical assembly parts. The software includes the automation for 1) calculating working dimensions from assigned stock removal final dimensions, 2) assigning working dimension tolerances, 3) calculating rough stock removal tolerances, 4) calculating resultant dimensions and tolerances, and 5) checking data and adjust chart.

Li et al. [5] have identified four phases which are required for an automatic tolerance analysis system: 1) automatic acquisition of all the related dimensions from a design database, 2) design assurance, 3) tolerance distribution, and 4) design-to-manufacturing coordinate translation.

\section{B. Modeling and Control of Geometric Tolerancing}

In order to comply with the ANSI geometric tolerance standards Y14.5, Wu and Zhang [6] convert these standards into representational primitives which can be unambiguously incorporated into a CAD system. Germani et al. [4] have developed a list representation approach to assist the analysis and computation during the generation of tolerance charts. Chen et al. [7] create an intuitionistic self-validation approach which use a robust method to dynamically compute the geometric relations for design features and update the theoretical properties of the relations.

Saravanan et al. [8] have generated an optimal tolerance design framework, which uses a matrix-tree-chain method to represent and identify critical operations. Irani et al. [3] have developed statistical models for analyzing position tolerance. The models can determine constraints inherited on production equipment as well as manufacturing processes in order to satisfy the cylindrical position tolerance if a drilled hole specified at regardless of feature size (RFS).

\section{Optimal Tolerance Assignment}

The tolerance optimization problems fall into two categories: the optimal tolerance assignment problem and the minimum cost problem. The objective for the first type of problem is to adjust the tolerance assignment of each critical operation in order to make the resultant tolerance be equal to the $\mathrm{B} / \mathrm{P}$ specification.

Wang et al. [9] have developed an iterative procedure to redistribute the residual tolerance based on the weighted factor pre-assigned to each operation. Tool wear effects are considered in the procedure but no process constraint is included. Li et al. [5] have also developed a two-stage heuristic to assign optimal tolerances by: 1) initially assigning a tolerance to every working dimension and 2) making necessary iterative adjustment to meet final needs. The initial tolerance is estimated based on an approximate method. The iterative procedure is invoked whenever there are excess tolerances or the stock removals are not sufficient for the cutting operations.

The objective for the second type of tolerance optimization problem is to distribute appropriate tolerance to each operation in order to minimize the processing cost. Chen et al. [7] develops a mathematical model to minimize the combined quality cost with a loss function and machining cost with a experienced-based fitted cost curve. Similar quality loss function approaches are observed in Haghighi et al. [1].

Saravanan et al. [8] have generated a minimum cost model by using mixed integer programming technique. Irani et al. [3] have suggested an improved cost model by incorporating machine capability constraints. However, the model ignores the machine setup constraints and selects machines by simply checking the machine capabilities. This assumption violates most of the engineering practices.

\section{CONCENTRICITY}

Besides the general linear dimension, tolerance control chart can also be applied to the geometric dimensioning, such as assembly parts, angular dimensions, heat-treatment, plating, polishing, chamfering, especially in calculating the concentricity [10].

The concentricity is a type of geometric location tolerancing. It is an important tolerance control feature for the hole/shaft machining operations. The concentricity is formed while two or more cylinders are produced about a common axis. The eccentricity, coaxiality, or more straight forward, the total deviation of two or more center lines can be represented by using concentricity tolerance. The 
concentricity is obtained by measuring the total runout of a surface about a datum. Normally, it is measured under the RFS principle but not the MMC condition. Please refer to Fig. 2 for graphic representation.

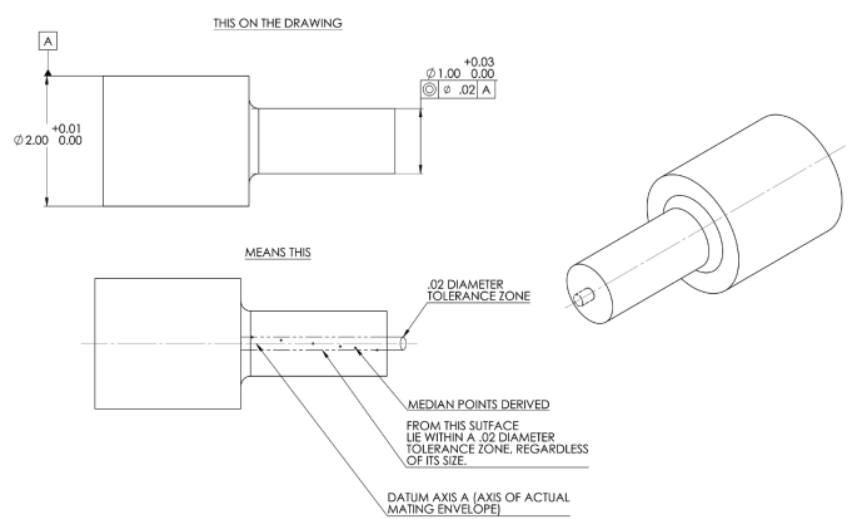

Fig. 2. Basic definition of concentricity [10].

\section{A. Manual Procedures for Preparing Concentricity Tolerance Chart}

The concentricity tolerance chart contains eight columns: 1) operation sequence number, 2) operation code, 3) machine, 4) operation description, 5) working tolerance, 6) tolerance bar working area, 7) balance tolerance, and 8) critical operations involved columns. An example of concentricity tolerance chart is given in Fig. 3. The first $\mathrm{B} / \mathrm{P}$ specification \pm 0.005 , is the concentricity between cylinders 1 and 2 and the second $\mathrm{B} / \mathrm{P}$ specification \pm 0.003 , is the concentricity between cylinders 2 and 3 . The procedures to calculate the resultant tolerance of the first $\mathrm{B} / \mathrm{P}$ specification are provided as follows:

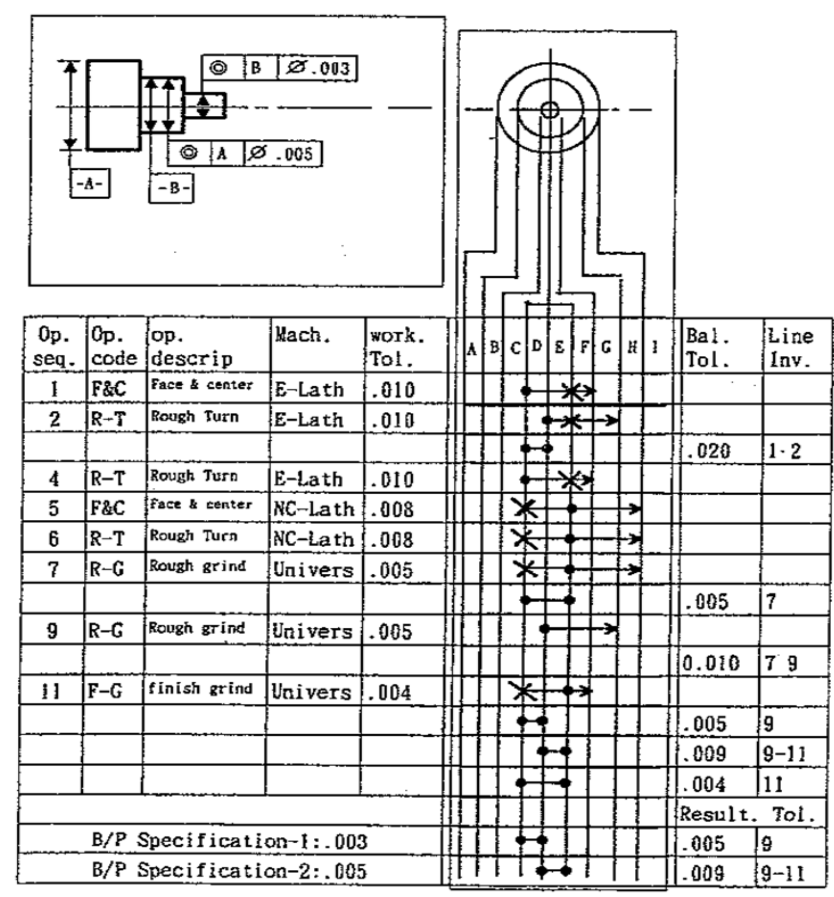

Fig. 3. Concentricity tolerance chart.

Step 1: Create a side view of the original part drawing.

1-1: Draw extension lines for all machining surfaces: A, B, $\mathrm{C}, \mathrm{G}, \mathrm{H}$, and I.

1-2: Create separate center lines for all cylinders: line D for cylinder 1, line $\mathrm{E}$ for cylinder 2, and line $\mathrm{F}$ for cylinder 3 respectively.

Step 2: Input the tentative process plan which has nine operations.

Step 3: Create a tolerance bar for each operation. The extension lines for the machining surfaces selected are to the right-hand-side of their center lines.

Step 4: Start tracing back from the right end of the resultant tolerance bar:

4-1: $\quad$ Trace upward until hitting the symbol of tolerance bar no. 12,

4-2: $\quad$ Trace along tolerance bar no. 12 until reaching the $\times$ symbol,

4-1: $\quad$ Trace upward until hitting the $\bullet$ symbol of tolerance bar no. 7 ,

4-2: $\quad$ Trace along tolerance bar no. 7 until reaching the $\times$ symbol,

4-1: $\quad$ Trace upward until hitting the symbol of tolerance bar no. 4 ,

4-2: $\quad$ Trace along tolerance bar no. 4 until reaching the $\times$ symbol,

4-1: $\quad$ Trace upward until hitting the symbol of tolerance bar no. 1 .

Step 5: Start tracing back from the left end of the resultant tolerance bar:

5-1: Trace upward until hitting the symbol of tolerance bar no. 11,

5-2: Trace along tolerance bar no. 12 until reaching the symbol,

5-3: Two tracing lines intersect with each other and fall into the same path.

Step 6: Calculate the resultant tolerance. The tolerance bars involved are no. 11 and no. 12. In other words, the critical operations are operation sequence no. 11 and no. 12. The accumulated tolerance is $\pm(0.004+0.004)= \pm 0.008$.

Step 7: Apply the same trace-back algorithm to $\mathrm{B} / \mathrm{P}$ specification no. 2 . The critical operation is operation sequence no. 12 and the resultant tolerance is \pm 0.004 . Step 8: Stop.

\section{B. Minimum Cost Approach for Tolerance Assignment of Concentricity Control}

The optimal tolerance assignment problem contains two phases: the first phase is to minimize the slack tolerance, i.e., the difference between the $\mathrm{B} / \mathrm{P}$ specification and the resultant tolerance, under the circumstance that the cost-tolerance model is not available. The second phase is to minimize the total cost by rearranging the tolerance assigned to each critical operation under the circumstance that the cost-tolerance model is available.

An analytical model for minimizing total processing cost by using mixed integer programming technique is developed which uses a linear cost-tolerance function to represents the relationship between cost vs. tolerance. The minimum cost model for concentricity is expressed as follows:

Objective Function:

$$
\operatorname{Min} \sum_{i=1}^{d} \sum_{l=1}^{e} \sum_{k=1}^{f}\left[C_{i k}+\left(h_{i k} \times t_{i}\right)\right] \times m_{i k} \times
$$

Subject to:

$$
L_{i k} \leq m_{i k} \leq \delta_{i l} \times t_{i} \leq U_{i k},
$$




$$
\sum_{i=1}^{d} \sum_{k=1}^{f} m_{i k} \leq \delta_{i l} \times t_{i} \leq b v_{l}, l=1,2, \cdots e
$$

where

$C_{i k}$ : operating cost for performing operation $i$ on machine $k$

$m_{i k}$ : machine $k$ performing operation $i$

$t_{i}$ : tolerance introduced by operation $i$

$L_{i k}$ : lower bound of the process capability for performing operation $i$ on machine $k$

$U_{i k}$ : upper bound of the process capability for performing operation $i$ on machine $k$

$h_{i k}$ : slope of the linear cost-tolerance function for performing operation $i$ on machine $k$

$\delta_{i l}:=1$, if $O_{i}$ is a critical operation to resultant tolerance wrt. the $l$ th $\mathrm{B} / \mathrm{P}$ specification

$b v_{l}$ : the variant portion of the $l$ th $\mathrm{B} / \mathrm{P}$ specification

Eq. (2) represents that the tolerances assigned to all operations should be complied with the constraints of process capabilities. Eq. (3) represents that the resultant tolerances should be less than or equal to the $\mathrm{B} / \mathrm{P}$ specifications. Unlike the minimum cost model for linear tolerance discussed in Irani et al. [3], the concentricity will not be affected by the amount of stock removal. Therefore, the constraint of stock removal tolerance does not exist in the minimum cost model for concentricity.

The example provided in Fig. 3 is applied to demonstrate the effectiveness for the Minimum Cost Approach. The operation with sequence number $9,\left(\mathrm{O}_{9}\right)$, is identified to be a critical operation to $\mathrm{B} / \mathrm{P}$ specification number $1,\left(b_{1}=\right.$ 0.003). Also, both of the operations with sequence number 9 and $11,\left(\mathrm{O}_{9}\right.$ and $\left.\mathrm{O}_{11}\right)$, are identified to be critical operations to $\mathrm{B} / \mathrm{P}$ specification number $2,\left(\mathrm{bv}_{2}=0.005\right)$. The values of operation significance factors are: $\delta_{9,1}=1, \delta_{9,2}=1$, and $\delta_{11,2}=1$. All the rest values of the operation significance factors are equal to zero.

For the Minimum Cost Approach, the process capability as well as the maximum operating cost for different machine assignment are given in Table I. Table II shows the results for four sets of process plans with the same operation sequence but different machine assignment. The optimal cost can be obtained for the first three plans while the slack tolerance are also minimized. The first process plan, to perform both rough and finish grinding operations on cylindrical grinder, has the lowest processing cost. The fourth process plan, to perform both rough and finish grinding operations on centerless grinder, has the highest processing cost but the slack tolerances are not minimized.

TABLE I: PROCESS CAPABILITY INFORMATION

\begin{tabular}{ccccc}
\hline & \multicolumn{2}{c}{ Cylindrical Grinder $\left(M_{1}\right)$} & \multicolumn{2}{c}{ Centerless Grinder $\left(M_{2}\right)$} \\
\cline { 2 - 5 } & $\begin{array}{c}\text { Rough } \\
\text { Grind }\left(O_{9}\right)\end{array}$ & $\begin{array}{c}\text { Finish } \\
\text { Grind }\left(O_{11}\right)\end{array}$ & $\begin{array}{c}\text { Rough } \\
\text { Grind }\left(O_{9}\right)\end{array}$ & $\begin{array}{c}\text { Finish } \\
\text { Grind }\left(O_{11}\right)\end{array}$ \\
\hline$L_{i k}$ & 0.003 & 0.002 & 0.002 & 0.001 \\
$U_{i k}$ & 0.010 & 0.009 & 0.008 & 0.006 \\
$h_{i k}$ & -0.43 & -1.29 & -0.83 & -2.0 \\
$C_{i k}$ & 0.08 & 0.12 & 0.15 & 0.20 \\
\hline
\end{tabular}

TABLE II: RESULTANT TOLERANCES AND MACHINING COST Process plans with different machine assignment
(1): $\left(O_{9}, M_{1}\right)$
(2): $\left(O_{9}, M_{1}\right)$
(3): $\left(O_{9}, M_{2}\right)$
(4): $\left(\mathrm{O}_{9}, M_{2}\right)$
$\rightarrow\left(O_{11}, M_{1}\right)$
$\rightarrow\left(O_{11}, M_{2}\right)$
$\rightarrow\left(O_{11}, M_{1}\right)$
$\rightarrow\left(O_{11}, M_{2}\right)$

Objective 0.28-0.86t $t_{1}-1.290 .36-0.86 t_{1}-2.0 \quad 0.42-1.66 t_{1}-1.290 .50-1.66 t_{1}-2.0$ Function $t_{2}$

\begin{tabular}{ccccc}
\hline$*$ & & & & \\
\hline$b v_{1}$ & 0.003 & 0.003 & 0.003 & 0.003 \\
$b v_{2}$ & 0.005 & 0.005 & 0.005 & 0.005 \\
$s v_{1}$ & -0.002 & -0.002 & -0.002 & -0.002 \\
$s v_{2}$ & -0.004 & -0.004 & -0.004 & -0.004 \\
$t_{1}^{*}$ & 0.003 & 0.003 & 0.003 & 0.002 \\
$t_{2}^{*}$ & 0.002 & 0.002 & 0.002 & 0.003 \\
$r v_{1}^{*}$ & 0.003 & 0.003 & 0.003 & 0.002 \\
$r v_{2}^{*}$ & 0.005 & 0.005 & 0.005 & 0.005 \\
$s v_{1}^{*}$ & 0 & 0 & 0 & 0.001 \\
$s v_{2}^{*}$ & 0 & 0 & 0 & 0 \\
\hline Total & 0.2748 & 0.3534 & 0.4124 & 0.4907 \\
Cost & & & & \\
\hline
\end{tabular}

$*: t_{1} \leq 0.003$ and $t_{1}+t_{2} \leq 0.005$

\section{CONTROL AND DESIGN ASPECTS OF COMPUTER-AIDED TOLERANCE CONTROL SYSTEM}

A computer-aided assembly process tolerance control system, is developed to automate the generation of concentricity tolerance charts and the assignment of optimal tolerance. The system is able to interface with any CAD system which can generate DXF files, such as AutoCAD from Autodesk, Inc. [11], and extract minimum information required to calculate the resultant tolerance for the proposed process plan and construct the tolerance chart. The system also provides a tolerance reassigning function which allows users to assign different weight to each critical assembly operation without changing the operation sequence and to optimize the slack tolerance. A user-friendly, full screen editor is developed to create, modify, delete, and review the process plan.

\section{A. Modular System Design}

The computer-aided assembly process tolerance control system is composed of five important modules. The first module is the CAD and dimension/tolerance information transformation module which extracts minimum design information from the engineering drawing created by AutoCAD. The second module is a full screen process plan editor which allows the user to input tentative process plans. The third module is a concentricity tolerance chart generator which first creates a simplified part drawing to represent the original design then construct the concentricity tolerance chart.

Similar to the concentricity tolerance chart generator, the last module is an optimal tolerance assignment module which allows the user to change the weights of critical operations and minimize the slack tolerances. The validated process plan then is saved into the process plan database for interfacing with the CAPP system.

\section{B. Generation of Design Drawings}

The first problem should be faced while developing a fully automated tolerance control chart system is how to generate design drawings, and link the CAD system to the tolerance control chart system. In other words, the question will become how to convert a CAD drawing data file into a compact format which can be directly processed by the automated tolerance chart software. Considering the popularity and compatibility, a general purpose CAD software, AutoCAD, by Autodesk, Inc., is chosen as a tool to generate the design drawings.

In order to differentiate the basic geometric data created in 
the Cartesian Coordinates with the dimension/tolerance specifications as well as other auxiliary text information, the design drawing is created under a stratified manner by storing design information into different layers. After the design drawing has been completed, the DXFOUT command of AutoCAD is used to convert the binary code data file into ASCII code data file which is then accessible by the system directly.

\section{Extraction of Dimension and Tolerance Information}

The ASCII code design drawing data file generated by the DXFOUT command of AutoCAD is in a general format which contains not only the majority geometric information but also lots of auxiliary drawing information. For example, the HEADER section contains general information about the design drawings, such as default value of coordinates, angles and text mode. The TABLE section contains information about line types, layer specifications, text styles and view ranges. The BLOCKS section contains all user defined blocks information. Only the last section, ENTITIES, contains dimension and tolerance data required by the system, such as basic dimensions, datum locations, concentricity tolerance specifications.

A CAD data transformation subroutine, CADTRANS.EXE, is developed to filter the data in the first three sections out of the system. Then, it will translate all dimension and tolerance data in the ENTITIES section of each layer into a more comprehensive and compact style then stores these converted data into separated data files accordingly.

After the design drawing has been filtered and translated, the user can invoke either the concentricity tolerance chart generator to verify the resultant tolerances of the tentative process plan. The user can also invoke the tolerance reassignment module to optimize the slack tolerance.

\section{CONCLUSIONS}

The location tolerance and orientation tolerance are two important tolerance requirements for assembly and shaft/hole making processes. Algorithms for generation concentricity tolerance charts are developed to verify tentative process plans, to analyze the resultant tolerances, and to identify critical operations. A weighted tolerance approach is developed to minimize the slack tolerances. Also, an optimal cost approach is developed to minimize the total processing cost by assigning optimal tolerance to critical operations.

A computer-aided assembly process tolerance control system, is developed to automate the generation of location and orientation tolerance charts as well as the weighted tolerance assignment algorithm. An off-line procedure is also developed for the minimum cost approach by using IBM ILOG CPLEX, a computer software can solve mixed integer programming problems. The verified process plans are saved into the process plan database which is accessible to the CAPP system and can be integrated into a CIM system. Besides the efficiency, the computer-aided assembly process tolerance control system also provides a user-friendly interface to construct tolerance charts.

\section{REFERENCES}

[1] P. Haghighi, P. Mohan, J. J. Shah, and J. K. Davidson, "Automatic detection and extraction of tolerance stacks in mechanical assemblies," in Proc. ASME 2014 International Design Engineering Technical Conferences and Computers and Information in Engineering Conference, 2014.

[2] G. Kaisarlis, C. Provatidis, and M. Sfantsikopoulos, "A new approach for the effectiveness of coaxiality tolerance verification," International Journal of Applied Science and Technology, vol. 4, no. 3, pp. 39-49, 2013.

[3] S. A. Irani, R. O. Mittal, and E. A. Lehtihet, "Tolerance chart optimization," International Journal of Production Research, vol. 27, no. 9, pp. 1531-1552, 1989.

[4] M. Germani, F. Mandorli, M. Mengoni, and R. Raffaeli, "CAD-based environment to bridge the gap between product design and tolerance control," Precision Engineering, vol. 34, no. 1, pp.7-15, 2010.

[5] H. Li, H. Zhu, X. Zhou, P. Li, and Z. Yu, "A new computer-aided tolerance analysis and optimization framework for assembling processes using DP-SDT theory," The International Journal of Advanced Manufacturing Technology, vol. 86, iss. 5, pp 1299-1310, 2016.

[6] Y. Wu and G. Zhang, "Tolerance mathematical model based on the variation of control points of geometric element," Journal of Mechanical Engineering, vol. 5, no.3, pp. 21-26, 2013.

[7] H. Chen, S. Jin, Z. Li, and X. Lai, "A modified method of the unified Jacobian-Torsor model for tolerance analysis and allocation," International Journal of Precision Engineering and Manufacturing, vol. 16, no. 8, pp. 1789-1800, 2015.

[8] A. Saravanan, C. Balamurugan, K. Sivakumar, and S. Ramabalan, "Optimal geometric tolerance design framework for rigid parts with assembly function requirements using evolutionary algorithms," International Journal of Advanced Manufacturing Technology, vol. 73, iss. 9-12, pp. 1219-1236, 2014.

[9] H. Wang, N. Pramanik, U. Roy, R. Sudarsan, R. D. Sriram, and K. W. Lyons, "A scheme for transformation of tolerance specifications to generalized deviation space for use in tolerance synthesis and analysis," in Proc. ASME 2002 International Design Engineering Technical Conferences and Computers and Information in Engineering Conference, 2002, pp. 1037-1045.

[10] NADCA, NADCA Product Specification Standards for Die Casting, $9^{\text {th }}$ ed., NADCA, Arlington Heights, Illinois, 2015, pp. 5-13.

[11] Autodesk, AutoCAD Users' Manual, Autodesk, Inc., Sausalito, CA., 2016.

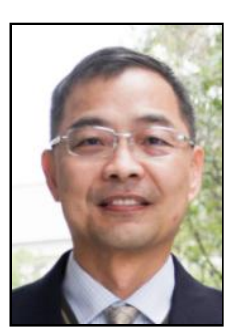

Chun-Wei R. Lin had his Ph.D. in industrial engineering from the Pennsylvania State University, USA in 1993, a MS degree in industrial management from the Oklahoma State University, USA in 1986, and a BS degree in industrial engineering from the National Tsing Hua University, Taiwan in 1982. He is currently Director of Creative Leadership Center, Distinguished Professor of Business Administration, and was Dear of Academic Affairs in Asia University, Taiwan. He is the a founding board member of Asia Pacific Business Innovation and Technology Management Association, board member of the Asia Pacific Industrial Engineering \& Management Systems Association, founding directors of Center for Innovation and Incubation for Small and Medium Enterprises and Center for Commercial Automation, YunTech, Taiwan. His major research and consulting interests are in intelligent production and process planning, green logistics management, enterprise resource planning, and health service industry management.

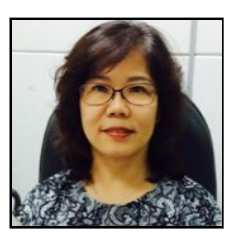

Yuh-Jiuan M. Parng had her Ph.D. in accounting from Yunlin University of Science and Technology, Taiwan, R.O.C. in 2010, and a MSA in accounting from University of Illinois at Chicago, USA. She is currently an Associate Professor of Accounting and Information Department at Asia University. Her major research and consulting interests are corporate compensation, and green accounting. 